\title{
Evaluation of different co-inoculation time of non-Saccharomyces/Saccharomyces yeasts in order to obtain reduced ethanol wines
}

\author{
María Victoria Mestre ${ }^{1,2, *, a}$, Yolanda Paola Maturano ${ }^{1,2, *}$, Laura Mercado ${ }^{3}$, María Eugenia Toro $^{1}$, Fabio Vazquez $^{1}$, \\ and Mariana Combina ${ }^{2,3}$ \\ ${ }^{1}$ IBT-FI-UNSJ. San Martín 1109 (O) Capital-San Juan, Argentina \\ ${ }^{2}$ Consejo Nacional de Investigaciones Científicas y Tecnológicas (CONICET), Argentina \\ ${ }^{3}$ Centro de Estudios Enológicos-EEA-Mendoza-INTA, San Martín 3853 Luján- Mendoza, Argentina
}

\begin{abstract}
Decreasing ethanol content in wines has become one of the main objectives of winemakers in different areas of the world. The use of selected wine yeasts can be considered one of the most effective and simple tools. The aim of this study was to evaluate the effect of co-inoculation times of selected non-Saccharomyces/Saccharomyces yeasts on the reduction of ethanol levels in wines. Hanseniaspora uvarum BHu9, Starmerella bacillaris BSb55 and Candida membranaefasciens BCm71 were co-inoculate with Saccharomyces cerevisiae under fermentative conditions. Treatments assayed were: pure fermentations of S. cerevisiae BSc203 and non-Saccharomyces yeasts BHu9, BSb55 and BCm71; -co-fermentations: A-BHu9/BSc203; B-BSb55/BSc203 and C-BCm71/BSc203. These co-inoculations were carried out under mixed (simultaneous inoculation), and sequential conditions (non-Saccharomyces yeasts inoculated at initial time and S. cerevisiae at 48, 96 and $144 \mathrm{~h}$ ). Lower fermentative efficiencies were registered when BHu9 and BSb55 remained pure more time. Conversely, the conversion efficiency was reduced in co-inocula of $\mathrm{BCm} 71 / \mathrm{BSc} 203$, when both yeasts interact more time. Metabolites produced during all vinification processes were within acceptable concentration ranges according to the current legislations. Conclusion Time interaction during fermentation processes of non-Saccharomyces and Saccharomyces yeasts showed influence on ethanol production, and this effect would be dependent on the co-inoculated species.
\end{abstract}

\section{Introduction}

Decreasing ethanol content in wines has become one of the main objectives of winemakers in different areas of the world. The problem of high alcohol levels in wine is related in part to global warming, which results in faster grape ripening, as well as an imbalance between sugar accumulation and the phenolic maturity of berries [1]. Numerous viticultural and engineering strategies have been proposed to achieve this purpose [2-5]. The use of selected non-Saccharomyces wine yeasts can be considered one of the most effective and simple tools. It is known that several non-Saccharomyces yeasts have different respiro-fermentative regulatory mechanisms compared to $S$. cerevisiae [6]. These microorganisms can divert consumption of carbon sources toward products other than ethanol. In this context, the use of nonSaccharomyces wine yeasts could be attractive to reduce alcohol content in wine. However, most nonSaccharomyces yeasts are not able to consume all sugars present in grape must and, therefore, they need to be used as part of sequential, mixed or multistarter inoculation with $S$. cerevisiae strains [7-10]. For this reason, it is interesting to analyze the interval between the first and the second inoculation (sequential culture) and inoculation

\footnotetext{
a e-mail: victoria_mestref@hotmail.com

* Both authors contributed equally to this work
}

proportions (mixed cultures) due to possible interactions between Saccharomyces and non-Saccharomyces yeasts. Therefore, the aim of the present study was to evaluate the effect of co-inoculation times of non-Saccharomyces/ Saccharomyces yeasts to the reduce ethanol in wines.

\section{Materials and methods}

\subsection{Microorganisms}

Saccharomyces cerevisiae BSc203, Hanseniaspora uvarum BHu9, Starmerella bacillaris BSb55 and Candida membranaefasciens $\mathrm{BCm} 71$ isolated from different oenological environments were assayed. Strains were obtained from the Culture Collection of Autochthonous Microorganisms at the Biotechnology Institute (IBT), Faculty of Engineering-National University of San Juan, Argentina, and were identified by conventional morphological, physiological and biochemical procedures according to [11]. Furthermore, molecular identification was determined by 5.8S-ITS-RFLP analysis [12].

\subsection{Fermentations}

Pre-inoculum of all yeast strains were obtained by growing them in sterile $13^{\circ} \mathrm{Bx}$ grape juice at $26 \pm$ $1{ }^{\circ} \mathrm{C}$ during $24 \mathrm{~h}$, under aerobic conditions (shaking 
at $130 \mathrm{rpm})$. These yeasts were inoculated at $1 \times$ $10^{6}$ cells $/ \mathrm{mL}$ in $250 \mathrm{~mL}$ Erlenmeyer flasks with $175 \mathrm{~mL}$ of sterile grape juice $\left(24^{\circ} \mathrm{Bx}, \mathrm{pH} 3.8\right.$, total acidity $5.5 \mathrm{~g} / \mathrm{L}$, fructose: $121 \mathrm{~g} / \mathrm{L}$ and glucose $117,2 \mathrm{~g} / \mathrm{L}$, nitrogen $200 \mathrm{mg} / \mathrm{L}$ ) and incubated at $24 \pm 1{ }^{\circ} \mathrm{C}$ under static conditions. Fermentation performance was monitored by $\mathrm{CO}_{2}$ released, measuring weight loss every $24 \mathrm{~h}$ during 21 days.

Treatments were: pure fermentations of non- Saccharomyces yeasts $\mathrm{BHu} 9, \mathrm{BSb} 55$ and $\mathrm{BCm} 71$ and $S$. cerevisiae $\mathrm{BSc} 203$ was used as control; -co-fermentations: - BHu9/BSc203; - BSb55/BSc203 and - BCm71/BSc203. These co-inoculations were carried out under mixed conditions (simultaneous inoculation 99\% non-Saccharomyce yeasts/ $1 \%$ S. cerevisiae): T1, and sequential conditions: non-Saccharomyces yeasts inoculated at initial time and $S$. cerevisiae at $48 \mathrm{~h}$ : T2, $96 \mathrm{~h}$ : T3 and $144 \mathrm{~h}$ : T4 (Table 1). Samples were taken at day 21. Ethanol, pH, volatile acidity, total acidity, density, glycerol, residual sugars, glucose, fructose, sucrose, lactic, malic, citric and tartaric acid were determined with a Bruker Wine-Scan infrared Fourier-transform spectrometer with Opus 7 software Version (ALPHA FT-IR Wine Analyzer).

\subsection{Statistical analysis}

Each assay was performed independently in triplicate and the results represent the average of the three determinations with the corresponding standard deviation $( \pm \mathrm{SD})$. Experimental data obtained during fermentations were analyzed by repeated analysis of variance (ANOVA) measurements. Significant differences were determined by Tukey's test and the results were considered significant if the associated $\mathrm{p}$ value was below 0.05. Spearman's correlation coefficients were calculated to determine statistical significance between ethanol and glycerol values of fermentation trials. SPSS version 19.0 was used for all tests.

\section{Results and discussion}

The use of non-Saccharomyces starters together with Saccharomyces cerevisiae in grape must fermentations has been investigated for more than 20 years [13-17] In this way, these studies have demonstrated that using non-Saccharomyces- S. cerevisiae yeasts represent a feasible way towards improving wine complexity and enhancing particular and specific characteristics of a wine [18] Therefore, these co-inoculation strategies provide better results than fermentations only inoculated with S. cerevisiae.

Recent studies have reported lower ethanol yields using co-cultures of non-Saccharomyces $-S$. cerevisiae yeasts [19-21]. However, possible interactions between different yeast species in mixed fermentations could influence on final ethanol levels and oenological traits in wines.

In previous experiments we have analyzed the oxidative-fermentative metabolism of 114 native nonSaccharomyces yeasts and their oenological traits in order to reduce ethanol in wines, when musts with high alcoholic potential are used. H. uvarum $\mathrm{BHu} 9$, S. bacillaris BSb55 and C. membranaefasciens $\mathrm{BCm} 71$

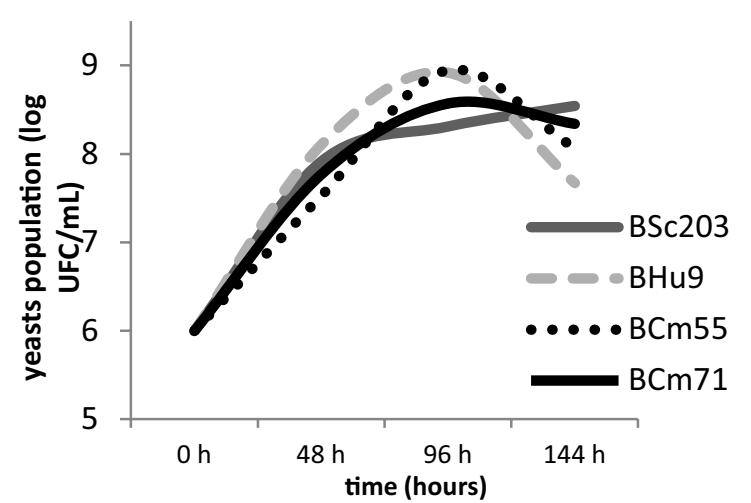

Figure 1. Evolution of non-Saccharomyces yeasts population $(\log \mathrm{CFU} / \mathrm{mL})$ previous to inoculation with Saccharomyces cerevisiae in treatments 4. BSc 203 was used as control.

were selected to design co-culture starters together with an autochthonous $S$. cerevisiae in lab-scale microvinification trials. Furthermore, we previously determined the killer phenomenon between non Saccharomyces and $S$. cerevisiae selected yeasts. All yeasts isolates showed neutral phenotype.

Analytical data from pure, mixed and sequential cultures of non- Saccharomyces/ Saccharomyces yeasts after 21 days of fermentation are presented in Table 2. We calculated conversion efficiency of sugars into ethanol (sugars consumption $(\mathrm{g} / \mathrm{L}) /$ ethanol produced $(\%)$ ) and we observed some trends. In the cases of BHu9-BSc203 and BSb55-BSc203 co-cultures, when non-Saccharomyces yeasts remained pure more time (T4: inoculation delays $144 \mathrm{~h}$ ), lower fermentative efficiencies were registered with values of $19.17 \mathrm{~g} / \%$ and $20.87 \mathrm{~g} / \%$, respectively. Furthermore, these co-inocula under mixed conditions showed similar efficiency conversion than $S$. cerevisiae control (Table 2). Several authors reported that a reduction in the alcohol level was achieved in fermentations using sequential inoculations [18-21]. In turn, these sequential inoculations showed better results when delay timing between non- Saccharomyces and S. cerevisiae yeasts was higher.

On the other hand, by the cases of BHu9 and BSb55 previous to inoculate with S.cerevisiae in treatments T4, their population started declining from the 96 hours (14\% and $10 \%$ respectively) (Fig. 1). This fact can be clearly related to nitrogen limitation, as was reported by Medina et al [22] who informed that non-Saccharomyces yeasts have an increased demand for nitrogen compared with S. cerevisiae. Therefore, we suggest to determinate the optimum time of supplementation of must with assimilable nitrogen source in order to allow the permanence of nonSaccharomyces yeasts and their consequent interaction with S.cerevisiae yeast inoculated.

Conversely, a different behavior was observed in the BCm71/ BSc203 co-inocula. The conversion efficiency was reduced $(20,28 \mathrm{~g} / \%)$ when both yeasts interact during longer time (Table 1). While in co-inocula with high delays (T3 and T4), we registered similar conversion efficiency than Saccharomyces cerevisiae BSc203 in pure conditions. Therefore, these results suggest that the products obtained during the fermentation process depended on possible interactions between co-participants yeasts. 
Table 1. Time inoculation (h) of non-Saccharomyces and S. cerevisiae co-cultures assayed.

\begin{tabular}{lccccccc}
\hline \multicolumn{7}{c}{ YEASTS ISOLATES } \\
\hline \multirow{2}{*}{ Treatment } & BHu9 & Sb55 & BCm71 & t0 & t48 & t96 & t144 \\
\hline T1 & $1 \times 10^{6}$ & $1 \times 10^{6}$ & $1 \times 10^{6}$ & $1 \times 10^{6}$ & & & \\
T2 & $1 \times 10^{6}$ & $1 \times 10^{6}$ & $1 \times 10^{6}$ & & $1 \times 10^{6}$ & & \\
T3 & $1 \times 10^{6}$ & $1 \times 10^{6}$ & $1 \times 10^{6}$ & & & $1 \times 10^{6}$ & \\
T4 & $1 \times 10^{6}$ & $1 \times 10^{6}$ & $1 \times 10^{6}$ & & & & $1 \times 10^{6}$ \\
\hline
\end{tabular}

References: Hanseniaspora uvarum BHu9, Starmerella bacillaris BSb55, Candida membranaefasciens BCm71, Saccharomyces cerevisiae BSc203 t0, t48, t96, t144: inoculation time of $S$. cerevisiae yeast (hours).

Table 2. Metabolic parameters of pure, mixed and sequential conditions of Hanseniaspora uvarum BHu9, Starmerella bacillaris BSb55 and Candida membranaefasciens BCm71 and S. cerevisiae BSc203

\begin{tabular}{|c|c|c|c|c|c|c|c|}
\hline & & Treatment & $\begin{array}{l}\text { Efficiency conversión } \\
\text { sugar }(g / L) / \text { ethanol }(\%)\end{array}$ & $\begin{array}{c}\begin{array}{c}\text { ethanol } \\
\text { production }\end{array} \\
(\% \mathrm{v} / \mathrm{v}) \\
\end{array}$ & $\begin{array}{c}\text { residual } \\
\text { sugars } \\
(\mathrm{g} / \mathrm{L})\end{array}$ & $\begin{array}{c}\begin{array}{c}\text { glycerol } \\
\text { production }\end{array} \\
(\mathrm{g} / \mathrm{L}) \\
\end{array}$ & $\begin{array}{l}\text { acetic acid production } \\
\qquad(\mathrm{g} / \mathrm{L})\end{array}$ \\
\hline \multirow{3}{*}{\multicolumn{2}{|c|}{ 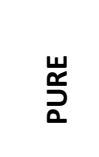 }} & BSc 203 & 17,04 & 13,9 & 0,5 & 9,2 & 0,62 \\
\hline & & BSb 55 & 19,92 & 11,9 & 1,1 & 10,4 & 0,57 \\
\hline & & $\mathrm{BCm} 71$ & 19,92 & 11,9 & 1,1 & 9,4 & 0,55 \\
\hline \multirow{13}{*}{ 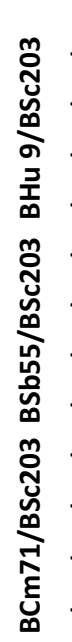 } & & B Hu9 & 18,00 & 12,2 & 18,6 & 9,3 & 0,57 \\
\hline & T1 & 99.1 & 17,47 & 13,2 & 7,6 & 8,3 & 0,65 \\
\hline & T2 & $48 \mathrm{H}$ & 16,93 & 13,4 & 8 & 8,4 & 0,6 \\
\hline & T3 & $96 \mathrm{H}$ & 19,53 & 12 & 3,8 & 9,4 & 0,7 \\
\hline & T4 & $144 \mathrm{H}$ & 19,17 & 12,3 & 2,4 & 10,4 & 0,61 \\
\hline & $\mathrm{T} 1$ & 99.1 & 17,98 & 13 & 4,4 & 8,3 & 0,59 \\
\hline & T2 & $48 \mathrm{H}$ & 19,14 & 12,4 & 1,1 & 9,6 & 0,55 \\
\hline & T3 & $96 \mathrm{H}$ & 17,05 & 13,2 & 13,1 & 9,1 & 0,58 \\
\hline & T4 & $144 \mathrm{H}$ & 20,89 & 11,4 & 0,9 & 9,7 & 0,5 \\
\hline & $\mathrm{T} 1$ & 99.1 & 20,28 & 11,7 & 0,9 & 9,8 & 0,55 \\
\hline & T2 & $48 \mathrm{H}$ & 19,50 & 12,1 & 2,2 & 9,2 & 0,51 \\
\hline & T3 & $96 \mathrm{H}$ & 19,24 & 12,3 & 1,6 & 9,1 & 0,54 \\
\hline & T4 & $144 \mathrm{H}$ & 17,52 & 13,3 & 5,2 & 8,8 & 0,56 \\
\hline
\end{tabular}

Specific antagonistic and synergistic interactions between different microorganisms may be found in food fermentations.

These interactions also play an important role in the succession of microbes in fermentation ecosystems. The work by Nissen [23] suggests that Quorum Sensing interactions may play a role in the interactions between yeast species in fermentation (possibly by cell-cell contact-mediated signaling or a diffusible signal). They demonstrated that the presence of a high concentration (107 cfu ml-1) of viable Saccharomyces arrested growth of Kluyveromyces thermotolerans and Torulaspora delbrueckii. According to these authors, the presence of ethanol and nutrient depletion was ruled out, but the cause of early growth arrest of non-Saccharomyces yeasts in mixed cultures was by a cell cell contact mechanism $[23,24]$. However, in the present study, at time inoculation of S.cerevisiae we detected higher levels population of non- Saccharomyces yeasts than S.cerevisiae (between $2 \times 10^{7}-3 \times 10^{8}$ vs. $1 \times 10^{6}$ in sequential cultures and $9,9 \times 10^{5}$ vs. $\times 10^{4}$ in mixed conditions). In this context, we expected that S.cerevisiae no arrested the growth of non- Saccharomyces yeasts (BHu9, BSb55 and BCm71), which gives them a competitive edge that allows their presence and permanence during alcoholic fermentation.

We evaluated the relationship between glycerol production and ethanol formation in the co-inoculated fermentations assayed. Results showed an inverse correlation between both final products (Spearman's coefficients of $-0,74, p=0.005)$. Thus, those treatments that registered less ethanol values present more glycerol values. Therefore, we could infer that under these conditions, non- Saccharomyces yeasts assayed divert its metabolic pathway toward glycerol-pyruvic fermentation. In spite of that glycerol and acetic acid are produced by the same metabolic pathway [25], all treatments registered low acetic acid values (lower than $0.7 \mathrm{~g} / \mathrm{L}$ ). In turn, acid acetic content of all treatments did not present significative differences $(\mathrm{p}=0.12)$ (Table 2).

In Table 3 we reported the analytical data of the 3 treatments selected. As we can observe, these treatments registered metabolites concentrations and values within acceptable ranges according to the current legislations. 
Table 3. Final wine parameters of 3 selected treatments.

\begin{tabular}{|c|c|c|c|}
\hline \multirow[b]{2}{*}{ ARAMETERS } & \multicolumn{3}{|c|}{ TREATMENTS } \\
\hline & 9/203-T4 & 55/203-T4 & 71/203-T1 \\
\hline ethanol (g/L) & 12.3 & 11.4 & 11.7 \\
\hline glycerol (g/L) & 8.3 & 8.3 & 9.7 \\
\hline acetic acid (g/L) & 0.61 & 0.5 & 0.55 \\
\hline fructose $(g / L)$ & 1.3 & 0.9 & 1.5 \\
\hline glucose $(\mathrm{g} / \mathrm{L})$ & 1.3 & 1 & 1 \\
\hline citric acid (g/L) & 0.48 & 0.41 & 0.25 \\
\hline density & 0.9952 & 0.9998 & 0.9962 \\
\hline malic acid (g/L) & 1.3 & 0.8 & 1.5 \\
\hline lactic acid (g/L) & 0.42 & 0.55 & 0.54 \\
\hline tartaric acid (g/L) & 1.68 & 1.52 & 1.77 \\
\hline pH & 3.13 & 3.22 & 3.14 \\
\hline total acidity (g/L) & 5.6 & 4.6 & 5.5 \\
\hline
\end{tabular}

In conclusion, non-Saccharomyces and Saccharomyces yeasts time interaction during fermentations showed influence on ethanol production and this effect would be dependent on the co-inoculated species. These interactions between the two species during grape must/ wine fermentation should be further studied and deepened. As the industry moves toward using more mixed cultures, the understanding of how these cultures interact and how one strain contributes to fermentation and another does not will be important to future research.

\section{References}

[1] A.J. Rodrigues, T. Raimbourg, R. Gonzalez, P. Morales. LWT-Food Sci. Technol 65, 1038 (2016)

[2] K. Bindon, C. Varela, J. Kennedy, H. Holt, M. Herderich. Food Chem 138 1696-1705 (2013)

[3] L. M. Schmidtke, J. W. Blackman, S. O. Agboola. J Food Sci 77, 25-41 (2012)

[4] H. R. Schultz \& M. Stoll. Aust. J. Grape Wine Res 16 4-24 (2010)

[5] S. Heux, J. M. Sablayrolles, R. Cachon, \& S. Dequin. Appl Environ Microbiol 72 5822-5828 (2006)

[6] M. Quirós, V. Rojas, R. Gonzalez, P. Morales. Int J Food Microbiol 18185 (2014)

[7] M. Ciani, F. Comitini, I. Mannazzu, P. Domizio. FEMS Yeast Res, 10, 123-133 (2010)
[8] T. Herraiz, G. Reglero, M. Herraiz, P. J. MartinAlvarez, M. D. Cabezudo. Am J Enol Vitic 41 313-318 (1990)

[9] N. P. Jolly, O. P. H. Augustyn, I. S. Pretorius. S Afr J Enol Vitic 24 55-62 (2003)

[10] R. Zironi, P. Romano, G. Suzzi, F. Battistutta, G. Comi. Biotechnol Lett 15, 235-238 (1993)

[11] S. A. Meyer, R. W. Payne, D. Yarrow, C. P. Kurtzman, J. W. Fell. The yeasts: a taxonomic study (1998)

[12] B. Esteve-Zarzoso C., Belloch, F. Uruburu, A. Querol. Int J Syst Bacteriol 49, 1, 329-337 (1999)

[13] M.E.Toro, F. Vazquez.World. J Microbiol Biotechnol 18, 4 (2002)

[14] M. Ciani, L. Beco, F. Comitini. Int J Food Microbiol 108 239-245 (2006)

[15] Y.P. Maturano, L.A. Rodriguez Assaf, M.E. Toro, M.C. Nally, M. Vallejo, L.I Castellanos de Figueroa, Vazquez F. 2012. Int J Food Microbiol 155, 43-50 (2012)

[16] Y. P. Maturano, M. V. Mestre, B. Esteve-Zarzoso, M. C. Nally, M. C. Lerena, M. E. Toro, M. Combina. Int J Food Microbiol 199, 23-32 (2015)

[17] M. Ciani \& F. Comitini. Ann Microbiol. 61,1 25-32 (2011)

[18] V. Englezos, K. Rantsiou, F. Cravero, F. Torchio, A. Ortiz-Julien, V. Gerbi, L. Cocolin). Appl Microbiol Biotechnol, 1-12 (2016)

[19] A. Contreras, C. Hidalgo, S. Schmidt, P. A. Henschke, C. Curtin, C. Varela. Int J Food Microbiol 205, 7-15 (2015)

[20] S. Di Maio, G. Genna, V Gandolfo, G. Amore, M. Ciaccio, \&, D. Oliva. S Afr J Enol Vitic, 33, 80-7 (2012)

[21] M. Ciani, L. Canonico, L. Oro, F. Comitini. BIO Web of Conferences 3, 02015 (2014)

[22] K. Medina, E. Boido , E. Dellacassa, \&, F. Carrau. Int J Food Microbiol 157, 245-250 (2012)

[23] P. Nissen, D. Nielsen, N. Arneborg. Yeast 20, 331341 (2003)

[24] P. Nissen \& N. Arneborg. Arch Microbiol 180, $257-$ 263 (2003)

[25] P. Ribéreau-Gayon, D. Dubourdieu, B. Donèche, A. Lonvaud. The Microbiology of Wine and Vinifications 1, 1-51 (2006) 